\title{
Modeling and Sensitivity Analysis of Dynamical Transmission of Lassa Fever
}

\author{
Musibau Abayomi Omoloye ${ }^{1}$, Akeem Olatoye Sanusi ${ }^{2}$, Iyabo Olamide Sanusi ${ }^{3}$, \\ Titilope Funmilayo Aminu ${ }^{4}$ \\ ${ }^{1,2,4}$ Department of Statistics, ${ }^{3}$ Department of Mathematics, \\ ${ }^{1,2,4}$ School of Applied Science, Federal Polytechnic, Offa, Nigeria. \\ ${ }^{3}$ Osun State College of Education, Ilesa, Nigeria \\ Corresponding Author: Akeem Olatoye Sanusi
}

\begin{abstract}
Lassa fever is an acute viral disease which occurs across West Africa regions. In view of this a non-linear deterministic model was considered to study dynamics spread of Lassa fever. Existence and uniqueness of the model were determined and the basic reproduction number of the model was computed using nextgeneration matrix, and sensitivity analysis of the model was performed and shows that the most sensitive parameters to the dynamical spread of Lassa fever are human birth rate $\beta_{H}$, followed by average number of female partners acquired by susceptible male $c_{2}$, and transmission rate resulting from sexual interaction between infected male and susceptible female $\eta_{2}$ have the highest values. It concludes that average number of female partners acquired by susceptible male and sexual transmission rate between the infected male and susceptible female should be reduced.
\end{abstract}

Keywords: Existence and Uniqueness, disease free equilibrium, basic reproduction numbers and sensitivity analysis

\section{INTRODUCTION}

Lassa fever is an acute viral disease which occurs across West Africa regions. The disease was discovered in 1969 when two missionary nurses died in Nigeria. The virus was named after the town in Nigeria where it first occurred. The disease is a member of the virus family Arenaviridae, is a single-stranded RNA virus and is zoonotic in the sense that human become infected after contact with infected animals.

Lassa fever is known to be endemic in parts of west Africa such as Benin, Ghana, Sierra Leone, Liberia, Mali, Guinea and Nigeria; however, as well as other neighboring countries are also at risk of this disease. The reservoir, or host, of Lassa virus is a rodent known as the "multimammate rat". When mastomys rats are infected, this rodent is able to excrete the virus in urine and faeces for an extended period of time or for the rest of its life. Mastomys rodents breed frequently, produce large numbers of offspring, and are numerous in the savannas and forests of west, central, and east Africa. In addition, Mastomys readily colonize human homes and areas where food is stored. All of these factors contribute to the relatively efficient transmission of Lassa virus from infected rodents to humans. Sexual transmission of Lassa virus is another case, Lassa fever occurs in all age groups and both sexes. Persons who are at greatest risk are those living in rural areas where Mastomys are usually found, especially in communities with poor sanitation or crowded living conditions. Health workers are at risk when caring for Lassa fever patients in the absence of proper barrier nursing and infection prevention and control practices (Disease outbreak news, 27 May, 2017). 
Signs and symptoms of Lassa fever mostly occur within 1-3 weeks when the patient comes in contact with the virus. Approximately $80 \%$ of Lassa fever virus infections have mild symptoms and undiagnosed. Mild symptoms include slight fever, general malaise, weakness and headache. About 20\% of infected individuals, however, disease may progress to more serious symptoms including hemorrhaging (in gums, eyes, or nose, as examples), respiratory distress, repeated vomiting, facial swelling, pain in the chest, back, and abdomen, and shock. Neurological problems have also been described, including hearing loss, tremors, and encephalitis. Death may occur within two weeks after symptom onset due to multi-organ failure. The most common complication of Lassa fever is deafness. Various degrees of deafness occur in approximately one-third of infections, and in many cases hearing loss is permanent. As far as is known, severity of the disease does not affect this complication: deafness may develop in mild as well as in severe cases. Onuorah et al (2016), developed a Lassa fever model using the sex structure approach. Their model represents the transmission dynamics of the Lassa fever disease using a set of ordinary differential equations. Susceptible individuals, male/female can be infected via interaction with the active Reservoir (Mastomys Natelensis), and via sexual interaction with opposite sex. Incorporate the rate at which virus is shedding into the environment into Onuorah at al. (2016) work the following model is developed. Omoloye et al (2020) investigates the application of DTM to solve dynamic transmission of Lassa fever model in a population. The mathematical model was formulated using first order differential equation. Firstly, existence and uniqueness of the solution was determined to establish that the model is mathematically well posed for the application of DTM. Numerically, simulations were conducted to compare the results obtained by DTM and that of fourthorder Runge-Kutta method.

\section{MODEL FORMULATION}

The model is constructed by dividing the total human population $N_{H}$ into four mutually exclusive sub-populations, Susceptible Male ${ }^{S_{1}(t)}$, Infected Male $I_{1}(t)$ and Susceptible Female $S_{2}(t)$, Infected Female $I_{2}(t)$. Also, the total Natural Reservoir or host population denoted by $N_{R}$ is divided into dormant Reservoir host $R_{1}(t)$, active Reservoir host $R_{2}(t)$ and $V(t)$ denote the population of Lassa virus in the environment.

Model has the following basic assumption, humans are only recruiting into the susceptible class through birth rate, and susceptible individuals, male or female can be infected through interaction with the active reservoir and sexual interaction with opposite sex and the rate at which virus is shedding into the environment. The force of infection is given by a standard incidence as $\frac{\left(c_{1} \eta_{1}(1-\varepsilon \tau) I_{2}+\eta_{3} R_{2}+\eta_{5} V\right)}{N_{H}}$

Where $N_{H}$ is the total population and the constants, $\eta_{1}, \eta_{2}, \eta_{3}, \eta_{4}$ and $\quad \eta_{5}$ is the transmission rate result from sexual interaction between either through susceptible female and infected male or susceptible male and infected female.

The model are described by the following differential equations

$$
\begin{aligned}
\frac{d S_{1}}{d t} & =\beta_{H} \theta N_{H}+\gamma I_{1}-\frac{\left(c_{1} \eta_{1}(1-\varepsilon \tau) I_{2}+\eta_{3} R_{2}+\eta_{5} V\right) S_{1}}{N_{H}}-\mu_{1} S_{1} \\
\frac{d I_{1}}{d t} & =\frac{\left(c_{1} \eta_{1}(1-\varepsilon \tau) I_{2}+\eta_{3} R_{2}+\eta_{5} V\right) S_{1}}{N_{H}}-\left(\mu_{1}+\delta_{1}+\gamma\right) I_{1} \\
\frac{d S_{2}}{d t} & =\beta_{H}(1-\theta) N_{H}+\gamma I_{2}-\frac{\left(c_{2} \eta_{2}(1-\varepsilon \tau) I_{1}+\eta_{4} R_{2}+\eta_{6} V\right) S_{2}}{N_{H}}-\mu_{1} S_{2} \\
\frac{d I_{2}}{d t} & =\frac{\left(c_{2} \eta_{2}(1-\varepsilon \tau) I_{1}+\eta_{4} R_{2}+\eta_{6} V\right) S_{2}}{N_{H}}-\left(\mu_{1}+\delta_{1}+\gamma\right) I_{2} \\
\frac{d R_{1}}{d t} & =\beta_{R} N_{R}-\left(\sigma+\mu_{2}+\delta_{2}\right) R_{1} \\
\frac{d R_{2}}{d t} & =\sigma R_{1}-\left(\mu_{2}+\delta_{2}\right) R_{2} \\
\frac{d V}{d t} & =e_{3} R_{2}+e_{2} I_{2}+e_{1} I_{1}-\phi V
\end{aligned}
$$


Table 1: Parameters used in the model

\begin{tabular}{|c|c|}
\hline Parameter & Description \\
\hline$\beta_{H}$ & Natural birth rate for human population \\
\hline$\beta_{R}$ & Natural birth rate for host population \\
\hline$\theta$ & Fraction of male birth $0<\theta<1$ \\
\hline$\eta_{1}$ & Transmission rate resulting from sexual interaction between infected female and susceptible male \\
\hline$\eta_{2}$ & Transmission rate resulting from sexual interaction between infected male and susceptible female \\
\hline$\eta_{3}$ & Transmission rate resulting from interaction between active virus Reservoir and susceptible male \\
\hline$\eta_{4}$ & Transmission rate resulting from interaction between active virus Reservoir and susceptible female \\
\hline$\eta_{5}$ & Transmission rate resulting from interaction between susceptible male with lassa virus in the environment \\
\hline$\eta_{6}$ & Transmission rate resulting from interaction between susceptible female with lassa virus in the environment \\
\hline$c_{1}$ & Average number of male partners acquired by a susceptible female \\
\hline$c_{2}$ & Average number of female partners acquired by a susceptible male \\
\hline$\mu_{1}$ & Natural death rate for human \\
\hline$\mu_{2}$ & Natural death rate for human \\
\hline$\gamma$ & Recovery rate of infected human \\
\hline$\sigma$ & Progression rate from dormant to active reservoir host \\
\hline$\delta_{1}$ & Death rate of human due to infection \\
\hline$\delta_{2}$ & Death rate of virus reservoir due to pesticide application \\
\hline$\varepsilon$ & Efficacy of condom \\
\hline$\tau$ & Compliance of condom \\
\hline$e_{1}$ & Discharging rate of lassa virus into the environment from infected male \\
\hline$e_{2}$ & Discharging rate of lassa virus into the environment from infected female \\
\hline$e_{3}$ & Discharging rate of lassa virus into the environment from active Reservoir \\
\hline$\phi$ & The death rate of lassa virus \\
\hline
\end{tabular}

\section{EXISTENCE AND UNIQUENESS OF SOLUTION \\ Theorem 1 Let}

$x_{1}^{\prime}=f_{1}\left(x_{1}, x_{2}, \ldots, x_{n}, t\right), x_{1}\left(t_{0}\right)=x_{10}$

$x_{2}^{\prime}=f_{2}\left(x_{1}, x_{2}, \ldots, x_{n}, t\right), x_{2}\left(t_{0}\right)=x_{20}$

$x_{3}^{\prime}=f_{3}\left(x_{1}, x_{2}, \ldots, x_{n}, t\right), x_{3}\left(t_{0}\right)=x_{30}$

:

$x_{n}^{\prime}=f_{n}\left(x_{1}, x_{2}, \ldots, x_{n}, t\right), x_{n}\left(t_{0}\right)=x_{n 0}$

Suppose $D$ is the region in $(n+1)$ dimensional space (one dimension for $t$ and $n$ dimensions for the vector $x$ ). If the partial derivatives $\frac{\partial f_{i}}{\partial x_{j}}$ where $\quad i, j=1,2, \ldots n$ are continuous in $D=\left\{(x, t):\left|t-t_{0}\right| \leq a,\left|x-x_{0}\right| \leq b\right\}$, Then there is a constant $\delta>0$ such that there exists a unique continuous vector solution $\underline{x}=\left[x_{1}(t), x_{2}(t), x_{3}(t), \ldots x_{n}(t)\right]_{\text {in }} \quad$ the interval $\left|t-t_{0}\right| \leq \delta$.

Let:

$$
\begin{aligned}
& f_{1}=\frac{d S_{1}}{d t}=\beta_{H} \theta N_{H}+\gamma_{1}-\frac{\left(c_{1} \eta_{1}(1-\varepsilon \tau) I_{2}+\eta_{3} R_{2}+\eta_{5} V\right) S_{1}}{N_{H}}-\mu_{1} S_{1} \\
& f_{2}=\frac{d I_{1}}{d t}=\frac{\left(c_{1} \eta_{1}(1-\varepsilon \tau) I_{2}+\eta_{3} R_{2}+\eta_{5} V\right) S_{1}}{N_{H}}-\left(\mu_{1}+\delta_{1}+\gamma\right) I_{1} \\
& f_{3}=\frac{d S_{2}}{d t}=\beta_{H}(1-\theta) N_{H}+\gamma_{2}-\frac{\left(c_{2} \eta_{2}(1-\varepsilon \tau) I_{1}+\eta_{4} R_{2}+\eta_{6} V\right) S_{2}}{N_{H}}-\mu_{1} S_{2} \\
& f_{4}=\frac{d I_{2}}{d t}=\frac{\left(c_{2} \eta_{2}(1-\varepsilon \tau) I_{1}+\eta_{4} R_{2}+\eta_{6} V\right) S_{2}}{N_{H}}-\left(\mu_{1}+\delta_{1}+\gamma\right) I_{2} \\
& f_{5}=\frac{d R_{1}}{d t}=\beta_{R} N_{R}-\left(\sigma+\mu_{2}+\delta_{2}\right) R_{1} \\
& f_{7}=\frac{d R_{2}}{d t}=\sigma R_{1}-\left(\mu_{2}+\delta_{2}\right) R_{2} \\
& f_{8}=\frac{d V}{d t}=e_{3} R_{2}+e_{2} I_{2}+e_{1} I_{1}-\phi V
\end{aligned}
$$

$D=\left\{\left(S_{1}, I_{1}, S_{2}, I_{2}, R_{1}, R_{2}, V\right):\left|S_{1}-S_{10}\right| \leq a,\left|I_{1}-I_{10}\right| \leq b,\left|S_{2}-S_{20}\right| \leq c,\left|I_{2}-I_{20}\right| \leq d,\left|R_{1}-R_{10}\right| \leq e,\left|R_{2}-R_{20}\right|\right\}$

The above equation (2.3) has a 
unique

solution.

Proof

Partial derivative was evaluated at the origin thus: $\left.\frac{\partial f_{1}}{\partial S_{1}}\right|_{(0,0,0,0,0,0,0)}=-\mu_{1},\left.\frac{\partial f_{2}}{\partial S_{1}}\right|_{(0,0,0,0,0,0,0)}=0$, $\left.\frac{\partial f_{3}}{\partial S_{1}}\right|_{(0,0,0,0,0,0,0)}=0,\left.\frac{\partial f_{1}}{\partial S_{1}}\right|_{(0,0,0,0,0,0,0)}=0$,

$\left.\frac{\partial f_{1}}{\partial I_{1}}\right|_{(0,0,0,0,0,0,0)}=\gamma$

$\left.\frac{\partial f_{2}}{\partial I_{1}}\right|_{(0,0,0,0,0,0,0)}=-\left(\mu_{1}+\delta_{1}+\gamma\right)$,

$\left.\frac{\partial f_{3}}{\partial I_{1}}\right|_{(0,0,0,0,0,0,0)}=0,\left.\frac{\partial f_{7}}{\partial I_{1}}\right|_{(0,0,0,0,0,0,0)}=e_{1}$,

$\left.\frac{\partial f_{1}}{\partial S_{2}}\right|_{(0,0,0,0,0,0,0)}=0,\left.\frac{\partial f_{2}}{\partial S_{2}}\right|_{(0,0,0,0,0,0,0)}=0$,

$\left.\frac{\partial f_{3}}{\partial S_{2}}\right|_{(0,0,0,0,0,0,0)}=-\mu_{2},\left.\frac{\partial f_{4}}{\partial S_{2}}\right|_{(0,0,0,0,0,0,0)}=0$,

$\left.\frac{\partial f_{1}}{\partial I_{2}}\right|_{(0,0,0,0,0,0,0)}=0,\left.\frac{\partial f_{3}}{\partial I_{2}}\right|_{(0,0,0,0,0,0,0)}=\gamma$

$\left.\frac{\partial f_{4}}{\partial I_{2}}\right|_{(0,0,0,0,0,0,0)}=-\left(\mu_{1}+\delta_{1}+\gamma\right)$,

$\left.\frac{\partial f_{7}}{\partial I_{2}}\right|_{(0,0,0,0,0,0,0)}=e_{2}$,

$\left.\frac{\partial f_{1}}{\partial R_{1}}\right|_{(0,0,0,0,0,0,0)}=0,\left.\frac{\partial f_{2}}{\partial R_{1}}\right|_{(0,0,0,0,0,0,0)}=0$,

$\left.\frac{\partial f_{5}}{\partial R_{1}}\right|_{(0,0,0,0,0,0,0)}=-\left(\sigma+\mu_{2}+\delta_{2}\right)$,

$\left.\frac{\partial f_{6}}{\partial R_{1}}\right|_{(0,0,0,0,0,0,0)}=\sigma$,

$\left.\frac{\partial f_{1}}{\partial R_{2}}\right|_{(0,0,0,0,0,0,0)}=0,\left.\frac{\partial f_{2}}{\partial R_{2}}\right|_{(0,0,0,0,0,0,0)}=0$,

$\left.\frac{\partial f_{6}}{\partial R_{2}}\right|_{(0,0,0,0,0,0,0)}=-\left(\mu_{2}+\delta_{2}\right)$,

$\left.\frac{\partial f_{7}}{\partial R_{2}}\right|_{(0,0,0,0,0,0,0)}=e_{3}$, $\left.\frac{\partial f_{1}}{\partial V}\right|_{(0,0,0,0,0,0,0)}=0,\left.\frac{\partial f_{2}}{\partial V}\right|_{(0,0,0,0,0,0,0)}=0$,

$\left.\frac{\partial f_{3}}{\partial V}\right|_{(0,0,0,0,0,0,0)}=0,\left.\frac{\partial f_{7}}{\partial V}\right|_{(0,0,0,0,0,0,0)}=-\phi$,

Since $\left|\frac{\partial f_{i}}{\partial x_{j}}\right|, i . j=1,2, \ldots 7$

are continuous and bounded. Hence, follow Derrick and Grossman of theorem 1 above, equation (3) has a unique solution and the model (1) is mathematically well posed.

\section{DISEASE FREE EQUILIBRIUM POINT}

At steady-state solution of the system (1) is obtained by setting

$\frac{d S_{1}}{d t}=\frac{d I_{1}}{d t}=\frac{d S_{2}}{d t}=\frac{d I_{2}}{d t}=\frac{d R_{1}}{d t}=\frac{d R_{2}}{d t}=\frac{d V}{d t}=0$

Let $E_{0}$ denote the disease free equilibrium state of the model (1)

At disease free

$$
E_{0}=\left(S_{1}, I_{1}, S_{2}, I_{2}, R_{1}, R_{2}, V\right)=\left(\frac{\beta_{H} \theta N_{H}}{\mu_{1}}, 0 \frac{\beta_{H}(1-\theta) N_{H}}{\mu_{1}}, 0,0,0,0\right)
$$

BASIC REPRODUCTION NUMBER

The basic reproduction number is the number of secondary cases of infection emanating from a single infection source[ Diekmann, O., Hesterbeek, J. A. P. and Metz, J. A. J.(1990], using next Generation matrix method to obtain the basic reproduction number. The matrices $\mathrm{F}$ (new infection terms) and $\mathrm{V}$ (other remaining transfer terms) are given as;

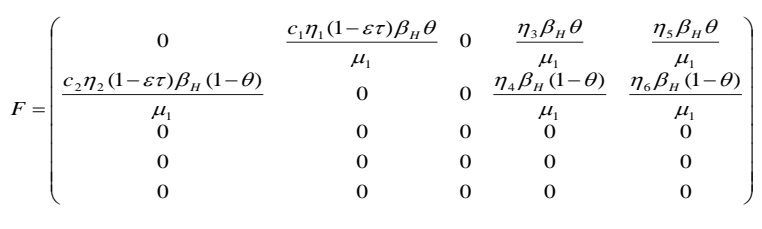

$V=\left(\begin{array}{ccccc}\mu_{1}+\delta_{1}+\gamma & 0 & 0 & 0 & 0 \\ 0 & \mu_{1}+\delta_{1}+\gamma & 0 & 0 & 0 \\ 0 & 0 & \sigma+\mu_{2}+\delta_{2} & 0 & 0 \\ 0 & 0 & & \mu_{2}+\delta_{2} & 0 \\ -e_{1} & -e_{2} & 0 & -e_{3} & \phi\end{array}\right)$

reproduction of model (1), denoted by $R_{0}$ is given by 


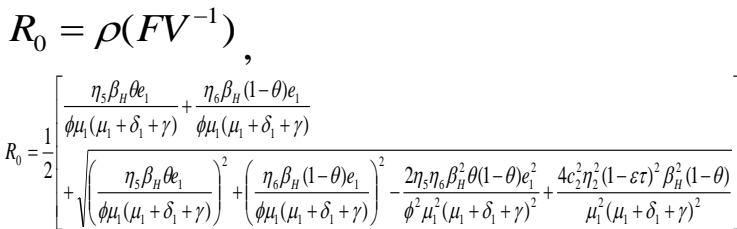

GLOBAL STABILITY OF DISEASE FREE EQUILIBRIUM

Global stability of epidemiological model is the most important and makes the model predictable as it guarantees that the model is independent of the initial size of the population this is achieved through (Castilo- Chavez et al 2002).

\section{Theorem 2}

The disease free equilibrium of the model (1) is Globally Asymptotically stable (GAS) if $R_{0}<1$

Proof

In establish the global stability of the disease free equilibrium, the two conditions (H1) and (H2) as in Castilo- Chavez et al 2002) must be satisfied for $R_{0}<1$ the model in (1) write in the form

$$
\begin{aligned}
& \frac{d X_{1}}{d t}=F\left(X_{1}, X_{2}\right) \\
& \frac{d X_{2}}{d t}=G\left(X_{1}, X_{2}\right) ; G\left(X_{1}, 0\right)
\end{aligned}
$$

Where $X_{1}=(x, z, v, w$,$) and X_{2}=(y, u, p)$

Component of $X_{1} \in R^{4}$ denoting uninfected population and the component of $X_{2} \in R^{3}$ denoting infected population.

From

DFE

$$
E_{0}=\left(X_{1}^{*}, 0\right), X_{1}^{*}=\left(\frac{\beta_{H} \theta N_{H}}{\mu_{1}}, 0 \frac{\beta_{H}(1-\theta) N_{H}}{\mu_{1}}, 0,0,0,0\right)
$$

Now, the first component is globally asymptotically stability of ${ }_{1}^{*}$, obtain

$$
\frac{d X_{1}}{d t}=F\left(X_{1}, 0\right)=\left[\begin{array}{c}
\beta_{H} \theta N_{H}-\mu_{1} x \\
\beta_{H}(1-\theta) N_{H}-\mu_{1} z \\
0 \\
0
\end{array}\right]_{(5)}
$$

From above equation (4), solve first order differential equation that is

$\frac{d x}{d t}+\mu_{1} x=\beta_{H} \theta N_{H}$

If integrating Factor I.F $=e^{\mu_{1} t}$, multiplying (6) by I.F obtain

$e^{\mu_{t} t}\left(\frac{d x}{d t}+\mu_{1} x=\beta_{H} \theta N_{H}\right) \Rightarrow e^{\mu_{1}} \frac{d x}{d t}+\mu_{1} x e^{\mu_{1} t}=\beta_{H} \theta N_{H} e^{\mu_{t} t}$

Write equation (7) as total differential

$\frac{d}{d t}\left(x e^{\mu_{1} t}\right)=\beta_{H} \theta N_{H} e^{\mu_{1} t}$

Integrate both sides of (8), obtain

$x e^{\mu_{1} t}=\frac{\beta_{H} \theta N_{H} e^{\mu_{1} t}}{\mu_{1}}+C$

$e^{\mu_{1} t}$ obtain

$x(t)=\frac{\beta_{H} \theta N_{H}}{\mu_{1}}+C e^{-\mu_{1} t}$

Applying $x(0)=0$, obtain divide through by $x(t)=\frac{\beta_{H} \theta N_{H}}{\mu_{1}}-\frac{\beta_{H} \theta N_{H}}{\mu_{1}} e^{-\mu_{1} t}$

Collect like term

$x(t)=\frac{\beta_{H} \theta N_{H}}{\mu_{1}}\left(1-e^{-\mu_{1} t}\right)$

Taking the limit as $t \rightarrow 0$, it gives

$$
x(t)=\frac{\beta_{H} \theta N_{H}}{\mu_{1}}
$$

Invariably,

$$
z(t)=\frac{\beta_{H}(1-\theta) N_{H}}{\mu_{1}}
$$

Hence,

$$
X_{1}^{*}=\left(\frac{\beta_{H} \theta N_{H}}{\mu_{1}}, 0 \frac{\beta_{H}(1-\theta) N_{H}}{\mu_{1}}, 0,0,0,0\right)_{\text {is }}
$$

globally asymptotically stable.

For second conditions that is

$$
G\left(X_{1}, X_{2}\right)=A X_{2}-G\left(X_{1}, X_{2}\right) \text { It gives }
$$




$$
A=\left(\begin{array}{lc}
-\left(\mu_{1}+\delta_{1}+\gamma\right) & \frac{n x}{N_{H}} \\
\frac{m z}{N_{H}} & -\left(\mu_{1}+\delta_{1}+\gamma\right) \\
0 & 0
\end{array}\right)
$$

Clearly, this is an M-matrix

$$
G\left(X_{1}, X_{2}\right)=\left(\begin{array}{ll}
\frac{\left(n u+\eta_{3} w+\eta_{5} p\right) x}{N_{H}} & -\left(\mu_{1}+\delta_{1}+\gamma\right) \\
\frac{\left(m y+\eta_{4} w+\eta_{6} p\right) z}{N_{H}} & -\left(\mu_{1}+\delta_{1}+\gamma\right) \\
e_{3} w-\phi p & e_{2}+e_{1}
\end{array}\right)
$$

Then, $\hat{G}\left(X_{1}, X_{2}\right)=A X_{2}-G\left(X_{1}, X_{2}\right) \geq 0$ $\hat{G}\left(X_{1}, X_{2}\right) \geq 0$ Since all the parameters are assumed to be non-negative,

This clearly $\hat{G}\left(X_{1}, X_{2}\right) \geq 0$ hence, this end proof.

\section{SENSITIVITY ANALYSIS}

Table 2: Sensitivity indices of reproduction number $R_{0}$ to parameters for the Lassa model

\begin{tabular}{|ll|}
\hline Parameter & Sensitivity indices \\
\hline$\beta_{H}$ & +1.00000001 \\
$c_{2}$ & +0.9735166 \\
$\eta_{2}$ & +0.9735166 \\
$e_{1}$ & +0.0264834 \\
$\eta_{6}$ & +0.01052437 \\
$\eta_{5}$ & +0.01595903 \\
$\theta$ & -1.46010237 \\
$\mu_{1}$ & -1.00179856 \\
$\gamma$ & -0.98021583 \\
$\tau$ & -0.08555899 \\
$\varepsilon$ & -0.08465362 \\
$\delta_{1}$ & -0.01798561 \\
$\phi$ & -0.0264834 \\
\hline
\end{tabular}

Sensitivity analysis is to assess the contribution and the relative impact of each of the parameters on basic reproduction number. The normalized forward sensitivity of the basic reproduction number with respect to all parameters tabulated in Table 3 is computed. The index allows us to measure the relative changes in a variable when a parameter changes.

The sensitivity index may be alternatively defined using partial derivatives. The normalized sensitivity index of $R_{0}$ that depends differentially on a parameter $p$ is

defined $y_{p}^{R_{0}}=\frac{\partial R_{0}}{\partial p} \times \frac{p}{R_{0}}$

\section{RESULT OF NUMERICAL SIMULATION}

Numerical simulation of the model was carried out by the help of MAPLE 18 software using the Runge-Kuta method of order four (4) together with parameter value given in the table 4 .

\begin{tabular}{|c|c|c|}
\hline Parameter & Values & References \\
\hline$\beta_{H}$ & 0.038 & CIA(2015) \\
\hline$\beta_{R}$ & 0.56 & Estimated \\
\hline$\eta_{1}$ & 0.6 & Estimated \\
\hline$\eta_{2}$ & 0.5 & Estimated \\
\hline$\eta_{3}$ & 0.5 & Estimated \\
\hline$\eta_{4}$ & 0.5 & Estimated \\
\hline$\eta_{5}$ & 0.5 & Estimated \\
\hline$\phi$ & 0.001 & Estimated \\
\hline$\tau$ & $0-1$ & Abdulrahaman(2014) \\
\hline$\sigma$ & 0.7 & Estimated \\
\hline$\delta_{1}$ & 0.2 & Estimated \\
\hline$\delta_{2}$ & 0.3 & Estimated \\
\hline$\mu_{1}$ & 0.02 & Estimated \\
\hline$\mu_{2}$ & 0.6 & Estimated \\
\hline$e_{1}$ & 0.01 & Estimated \\
\hline$\varepsilon$ & 0.8 & Garba\&Gumel(2010) \\
\hline$e_{2}$ & 0.7 & Estimated \\
\hline$e_{3}$ & 0.9 & Estimated \\
\hline$c_{1}$ & 2.0 & Williams et al (1996) \\
\hline$c_{2}$ & 3.0 & Williams et al (1996) \\
\hline
\end{tabular}

Table 3. Table of parameters and their values. 


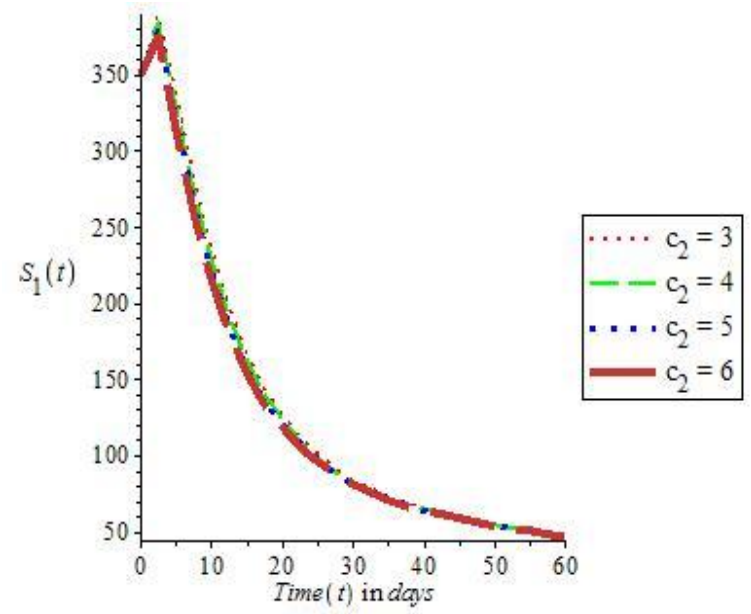

Fig 1: Show behaviours of Susceptible male

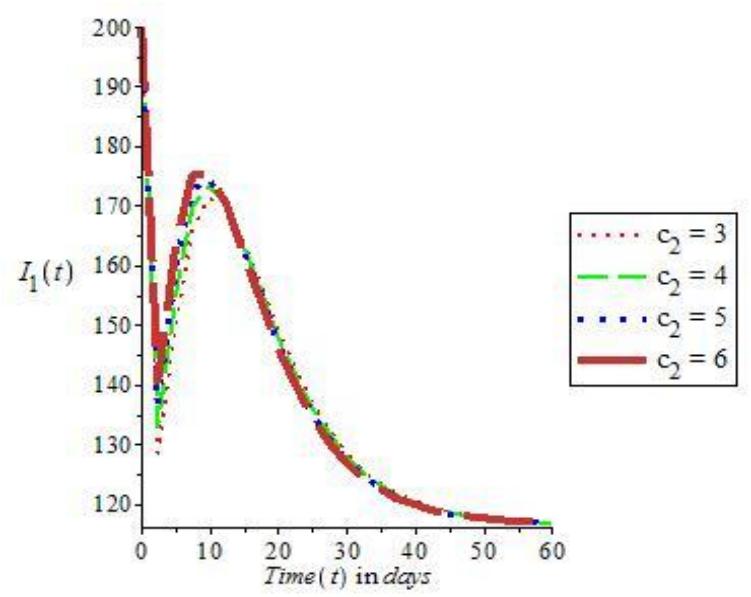

Fig 2: Show behaviours of Infected male

\section{DISCUSSION}

In figure 1 as the average number of female partner acquired increases, susceptible male population increases as a result of birth rate and recovery rate of infected male but later decreases as a result of increasing in female partner acquired. In figure 2 , infected male population decreases but later increases a little bit before finally decreases, this attributed to increasing in number of female partners acquired. Also, in figure 3 , initially susceptible female population increases but later decreases as a result of sexual transmission with infected male.

Finally, in figure 4 , infected female population decreases and increases as a result of sexual transmission.

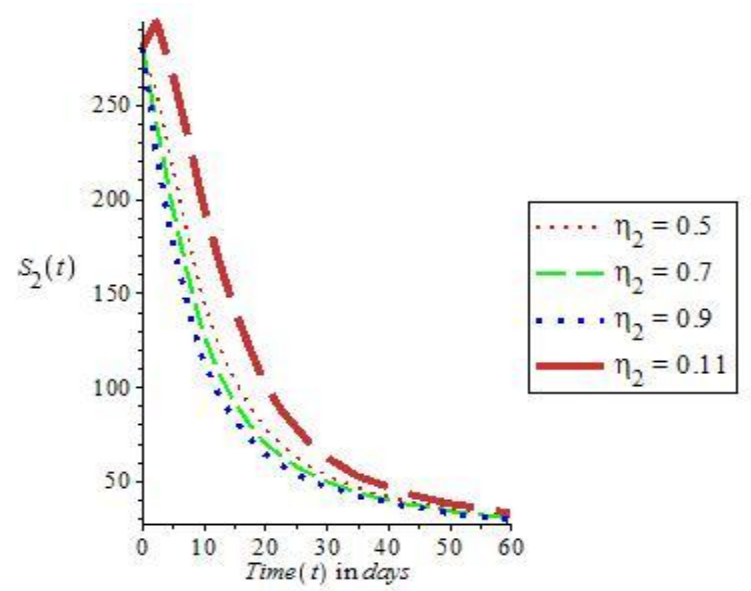

Fig 3: Show behaviours of Susceptible female

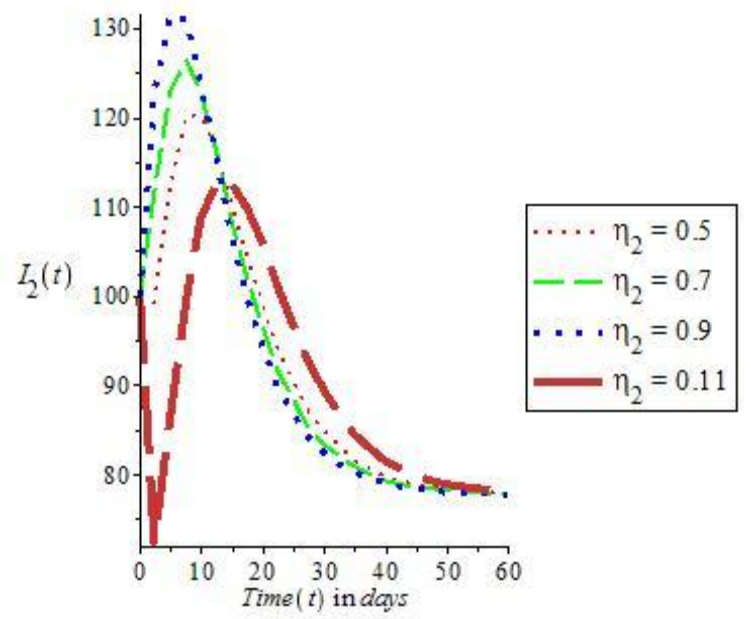

Fig 4: Show behaviours of Infected female

\section{CONCLUSION}

A mathematical model was developed and analyzed to gain more insight into the effect of transmission on dynamical spread of Lassa fever. Mathematically, the model consists of seven - nonlinear system of differential equation. It shows that there exists a domain $D$ where the model is mathematically and epidemiologically well posed. The basic reproduction number was determined, disease free equilibrium point was obtained and global stability of disease free equilibrium point is stable. Also from the table 3 above, the sensitivity analysis of the model reveals that the most sensitive parameters to the basic reproduction number $R_{0}$ are human birth rate, followed by average number of female partners acquired by susceptible male $c_{2}$ and transmission rate resulting from sexual interaction between 
infected male and susceptible female $\eta_{2}$ have the highest values. It concludes that the average number of female partners acquired by susceptible male and sexual transmission rate between the infected male and susceptible female should be reduced.

\section{Acknowledgement: None}

\section{Conflict of Interest: None}

\section{Source of Funding: None}

\section{REFERENCES}

1. Anderson, R. M. and May R. M. (1991). Infectious Diseases of Humans: Dynamics and Control. Oxford: Oxford University Press.

2. Bawa, M., Abdulrahaman, S., Jimoh, O.R., \& Adabara, N.U. (2013). Stability analysis of the disease-free equilibrium state of Lassa fever disease. Journal of Science and Technology, Mathematics and Education (JOSTMED), 9(2), 115 - 123.

3. Castill- Chavez C., Feng Z., \& Huang W. (2002). On the computation of $R_{0}$ and its role on global stability.

4. Centre for Disease Control. Imported Lassa fever. Morbidity Mortal Weekly Reports, 53(38), 894-897

5. Central Intelligence Agency (2015). World fact book for the year 2014. Retrieved on 20 February 2016 from http:/www.cia/library/publication/the-world fact book/geos/ni.htm.

6. Driessche, V. P., \& Wathmough, J. (2005). Reproductive Number and Sub-Threshold Endemic Eqilibria for Compartment Modelling of Disease Transmission. Mathematics Bioscience, 180, 29-48.

7. Eze, K. C., Salami T. A. T, Eze I. C., Pogoson, A. E., Omodia, N., \& Ugochukwu, M. (2010). High Lassa Fever Activity in Northern Part of Edo State Nigeria: Re Analysis of Confirmatory Test Result, African Journal of Health Sciences,16(34), 52-56.

8. Fisher-Hoch, S.P., Tomori, O., Nasidi, A., Perez-Oronoz, G.I., Fakile, Y., \& Hutwagner, L. (1995). Review of cases of nosocomial Lassa fever in Nigeria: the high price of poor medical practice. Biomedical Journal, 311, 857-869.
9. Garba M. S., Gumel, A.B., \& Abubakar, M.R. (2008), Backward Bifurcation in Dengue transmission dynamics. Mathematic al Bioscience Doi:10.1016/j.mbs.2008.05.00 2.

10. Garba, S. M., \& Gumel, A. B. (2010). Mathematical Recipe for HIV Elimination in Nigeria. Journal of the Nigeria Mathematical Society, 29, 51-112 International Journal of Mathematics and Statistics Studies Vol.4, No.1, pp.30-49, February 2016 Published by European Centre for Research Training and Development UK (www.eajournals.org)

11. Günther, S., Weisner, B., Roth, A., Grewing, T., Asper, M., Drosten, C., Emmerich, P., Petersen, J., Wilczek, M., \& Schmitz, H.(2001). Lassa Fever Encephalopathy: Lassa Virus in Cerebrospinal Fluid but Not in Serum. The Journal of Infectious Diseases, 184(3), 345-349. doi:10.1086/322033.2001. 11443561

12. Hethcote, H.W. (1978). An Immunization Model for Heterogeneous Population. Theoretical Population Biology 14(1978), 338-349

13. Lakshmikantham, V., Leela, S., \& Martynyuk, A. A. (1999). Stability Analysis of Non- linear systems, 164. New York and Basel: Marcel Dekker, Inc.

14. Okuonghae, D. \& Okuonghae, R. (2006). A Mathematical model for Lassa fever. Journal of the Nigerian Association of Mathematical Physics, 10,457-464.

15. Ogbu,O. E., Ajuluchukwu, C. J.,\& Uneke, C.J. (2007). Lassa fever in West Africa subregion: an Overview. Journal of Vector Borne Diseases 44, 1-11.

16. Ogabi, C.O., Olusa, T.V., \& Madufor, M.A. (2012). Controlling Lassa Fever in Northern Part of Edo State, Nigeria using SIR Model. New Science Journal 5(12), 115-121.

17. Omilabu, S.A, Badaru, S.O., Okokhere, P., Asogun, D., Drosten, C., \& Emmerich, P. (2005). Lassa fever, Nigeria, 2003 and 2004. Emerging Infectious Diseases, $11,1642-4$.

18. Onuorah, M .O., Ojo, M .S., Usman, D.J., Ademu, A.(2016) Basic Reproductive Number for the Spread and Control of Lassa fever, International Journal of Mathematics Trends and Technology (IJMTT). Vol. 30(1):1-7 
19. Omoloye, M.A., Yusuff, M. I. \& Emiola, O.K.S. Application of differential transformation method for solving dynamical transmission of Lassa fever model. World Academy of Science, Engineering and Technology International Journal of Physical and Mathematical Sciences Vol: 14, No: 11, 2020, Pp: 151154.

20. Onuorah, M.O, Akinwande, N.I,Nasir, M.O and Ojo, M.S, Sensitivity analysis of lassa fever model, International Journal of Mathematics and Statistics studies. Vol.4, No.1, Pp30-49,February 2016

21. Promed-mail. (2006). Lassa fever - Liberia (02). Number 20061001.2812. Retrieved on July 8, 2013 from http://www.promedma il.chip.org/pipermail/promed/003770.html

22. Richmond, J. K., \& Deborah, J. B. (2000). Lassa Fever Epidemiology, Clinical features, and Social Consequences. Biomedical Journal, 327, 1271-1275.

23. Tara, K. H. (2004). Virology notes in Lassa fever. Retrieved on March 10, 2012 from www.taraharper.com/v lass.html World Health Organisation. (2004).

How to cite this article: Omoloye MA, Sanusi AO, Sanusi IO et.al. Modeling and sensitivity analysis of dynamical transmission of lassa fever. International Journal of Research and Review. 2021; 8(10): 531-539. DOI: https://doi. org/10.52403/ijrr.20211067

$* * * * * *$ 